\title{
Is it possible to monitor nanoplastic's degradation through carbon isotope fingerprinting?
} WICKMANN $^{1}$, ALINE DIA ${ }^{1}$, CHRISTELLE ANQUETIL ${ }^{2}$ AND SYLVIE DERENNE ${ }^{2}$

${ }^{1}$ University of Rennes 1

${ }^{2}$ Sorbonne University

Presenting Author: anne-catherine.pierson-wickmann@univrennes1.fr

Plastic production has increased dramatically over the past century. This has resulted in a generation of plastic waste. Such plastic pollution encountered in marine or continental environments occurs in the form of macro-plastics, microplastics or even nanoplastics (NPs) depending on their size.

Recent studies have shown that NPs are essentially produced in aquatic systems by fragmentation of macro- and microplastics. As reactive nanoparticles, they can also be vectors for contaminants, and then spread into aquatic systems. Despite growing awareness of the problem, the environmental impact of NP pollution remains poorly understood. The development of remediation techniques requires to understand their reactivity variation due to their state of degradation. Indeed, NPs continue to degrade in the environment, chiefly related to UV radiation, mechanical stirring, biological activity. The degradation of polymer chains and surface functions generate changes in the chemical structure, inducing reactivity change issues.

Although a previous study evidenced that carbon stable isotope fingerprinting could be used to characterize plastics on a macroscopic scale, there was still a lack of knowledge about any possible extrapolation to the nanoscale.

In this context, degradation of NP standards was carried out using an UV reactor to mimic photo-degradation. The monitoring of the $\mathrm{d}^{13} \mathrm{C}$ signature during NP degradation was coupled with that of the morphology by Transmission Electron Microscopy (TEM), the size by the mean of Dynamic Light Scattering (DLS) and the chemical composition by Pyrolysis connected to Gas Chromatography coupled to Mass Spectrometry (Py-GC-MS).

The results showed: (i) a sharp increase in the isotopic ratio of $\mathrm{d}^{13} \mathrm{C}$ during the degradation, (ii) an evolution of the morphology and (iii) a substantial variation in the chemical composition

This first study on the ability of $\mathrm{d}^{13} \mathrm{C}$ signature to track NP degradation provides a key assessment that stable $\mathrm{C}$-isotopes can be promising tools for identifying and monitoring degradation pathways of NPs in the environment. As such, this study imposes additional constraints on the degradation processes and how they affect NPs along with their environmental dissemination, which is an issue on its own but also, potentially, through other pollutants that they might transport and thus release or sequester. 